\title{
Use of rate control medication before cardioversion of recent-onset atrial fibrillation or flutter in the emergency department is associated with reduced success rates
}

Gabriel E. Blecher, PDM, MBBS(Hons), FACEM*; Ian G. Stiell, MD, MSc*; Brian H. Rowe, MD, MSc ${ }^{\dagger}$; Eddy Lang, MD; Robert J. Brison, MD, MPH ${ }^{\S}$; Jeffrey J. Perry, MD, MSc*; Catherine M. Clement, RN"; Bjug Borgundvaag, MD, PhD ; Trevor Langhan, $\mathrm{MD}^{\ddagger}$; Kirk Magee, MD, MSc" ${ }^{\#}$ Rob Stenstrom, MD, PhD**; David Birnie, MD ${ }^{\dagger+}$; George A. Wells, $\mathrm{PhD}^{\dagger+}$

\section{ABSTRACT}

Objective: It is believed that when patients present to the emergency department (ED) with recent-onset atrial fibrillation or flutter (RAFF), controlling the ventricular rate before cardioversion improves the success rate. We evaluated the influence of rate control medication and other variables on the success of cardioversion.

Methods: This secondary analysis of a medical records review comprised 1,068 patients with RAFF who presented to eight Canadian EDs over 12 months. Univariate analysis was performed to find associations between predictors of conversion to sinus rhythm including use of rate control, rhythm control, and other variables. Predictive variables were incorporated into the multivariate model to calculate adjusted odds ratios (ORs) associated with successful cardioversion.

Results: A total of 634 patients underwent attempted cardioversion: 428 electrical, 354 chemical, and 148 both. Adjusted ORs for factors associated with successful electrical cardioversion were use of rate control medication, 0.39 (95\% confidence interval [Cl] 0.21-0.74); rhythm control medication, 0.28 (95\% Cl 0.15-0.53); and $\mathrm{CHADS}_{2}$ score > $0,0.43$ $(95 \% \mathrm{Cl} 0.15-0.83)$. ORs for factors associated with successful chemical cardioversion were use of rate control medication, 1.29 (95\% Cl 0.82-2.03); female sex, 2.37 (95\% Cl 1.50-3.72); and use of procainamide, $2.32(95 \% \mathrm{Cl} 1.43-3.74)$.

Conclusion: We demonstrated reduced successful electrical cardioversion of RAFF when patients were pretreated with either rate or rhythm control medication. Although rate control medication was not associated with increased success of chemical cardioversion, use of procainamide was. Slowing the ventricular rate prior to cardioversion should be avoided.

\section{RÉSUMÉ}

Objectif: On croit que lorsqu'un patient se présente au service des urgences (SU) en état de fibrillation auriculaire (flutter) récente, le contrôle de la fréquence ventriculaire avant l'amélioration de la cardioversion augmente le taux de réussite. Nous avons évalué l'influence des médicaments de contrôle de la fréquence et d'autres variables sur l'amélioration de la cardioversion.

Méthodes: Cette deuxième analyse des dossiers médicaux porte sur 1068 patients en état de fibrillation auriculaire (flutter) récente qui se sont présentés dans huit services des urgences au Canada au cours des 12 derniers mois. Une analyse univariée a été effectuée afin d'établir des liens entre les prédicteurs de conversion et le rythme sinusal, y compris le contrôle de la fréquence, le contrôle du rythme, et d'autres variables. Les variables prédictives ont été intégrées au modèle à plusieurs variables afin de calculer les ratios d'incidence approchés (RIA) redressés associés à la réussite de la cardioversion.

Résultats: Au total, 634 patients ont fait l'objet d'une tentative de cardioversion: 428 cardioversions électriques,

From the *Department of Emergency Medicine, University of Ottawa, Ottawa, ON; †Department of Emergency Medicine, University of Alberta, Edmonton, AB; $¥$ Division of Emergency Medicine, University of Calgary, Calgary, AB; §Department of Emergency Medicine, Queen's University, Kingston, ON; IIClinical Epidemiology Program, Ottawa Hospital Research Institute, Ottawa; $\uparrow$ Division of Emergency Medicine, University of Toronto, Toronto, ON; \#Department of Emergency Medicine, Dalhousie University, Halifax, NS; **Department of Emergency Medicine, University of British Columbia, Vancouver, BC; +†University of Ottawa Heart Institute, University of Ottawa, Ottawa.

Correspondence to: Dr. Gabriel E. Blecher, Clinical Epidemiology Unit, F663, Ottawa Hospital, 1053 Carling Avenue, Ottawa, ON K1Y 4E9; gabyblecher@gmail.com.

This article has been peer reviewed. 
354 cardioversions pharmacologiques, et 148 cardioversions électriques et pharmacologiques. Les RIA modifiés en tenant compte des facteurs liés aux cardioversions électriques réussies étaient I'utilisation de médicaments de contrôle de la fréquence, 0,39 (intervalle de confiance [IC] de $95 \% 0,21-$ 0,74 ); les médicaments de contrôle du rythme, 0,28 (IC $95 \%$ $0,15-0,53$ ); et les cotes $\mathrm{CHADS}_{2}>0,0,43$ (IC $95 \% 0,15-0,83$ ). Les RIA pour les facteurs liés aux cardioversions pharmacologiques réussies étaient l'utilisation de médicaments de contrôle de la fréquence, 1,29 (IC 95 \% 0,82-2,03); le sexe féminin, 2,37 (IC $95 \%$ 1,50-3,72); et I'utilisation de la procaïnamide, $2,32(\mathrm{Cl} 95 \%$ 1,43-3,74).
Conclusion: Nous avons démontré un taux de réussite plus faible des cardioversions pour les patients en état de fibrillation auriculaire (flutter) récente lorsque ces derniers avaient préalablement pris des médicaments de contrôle de la fréquence ou du rythme. Les médicaments de contrôle de la fréquence n'étaient pas associés à un taux de réussite plus élevé des cardioversions pharmacologiques, contrairement à la procaïnamide. II faudrait éviter de ralentir la fréquence ventriculaire avant la cardioversion.

Keywords: atrial fibrillation, cardioversion, rate control
Atrial fibrillation (AF) affects an estimated 2.2 million Americans and is present in 8 to $10 \%$ of those over 80 years old. ${ }^{1}$ The major morbidity associated with AF or atrial flutter (AFL) is from thromboembolism, particularly to the cerebral circulation. In managing this risk, one of two competing management strategies is recommended: rate control or rhythm control.

Symptomatic AF or AFL with rapid ventricular response is a common presentation to emergency departments (EDs). Recent-onset atrial fibrillation or flutter (RAFF) is defined as first episode, recurrent paroxysmal or recurrent persistent, and time to presentation within 48 hours of symptom onset. In this setting, emergency physicians have the option of restoring sinus rhythm via electrical or chemical means. Alternatively, the rate control strategy involves slowing the ventricular rate and commencing anticoagulation in patients who are at increased embolic risk, with planned outpatient cardiology follow-up. Current evidence on the optimal strategy suggests no advantage of rhythm over rate control; however, these studies were not performed in the ED and so include few patients with recent-onset dysrhythmias. ${ }^{2,3}$

\section{IMPORTANCE}

It has been noted that evidence-based guidance on the ED management of RAFF is lacking. ${ }^{4}$ Much of the research and guidelines have been developed in, and have almost exclusively been applied to, the outpatient or postoperative environment. The most recent American College of Cardiology/American Heart Association/European Society of Cardiology (ACC/AHA/ESC) guidelines on AF are a case in point. ${ }^{5}$ Because cardiologists have performed the majority of work in determining factors predictive of successful cardioversion, the majority of RAFF has been excluded. Identifying which aspects of management influence successful cardioversion could lead to widespread uptake of those practices and abandonment of those that do not. Known patient factors associated with successful cardioversion include patient factors such as weight, ${ }^{6}$ duration of arrhythmia, ${ }^{7}$ and a history of hypertension. ${ }^{6}$ Treatment factors include those affecting skin impedance, electrode placement, biphasic versus monophasic waveform, and initial and total energy. ${ }^{7}$ Drugs that reduce the cardioversion threshold include ibutilide, sotalol, digoxin, and dofetilide. ${ }^{8}$

Some practitioners believe that slowing the ventricular response before attempting cardioversion increases the success rate, although there is little evidence to support this. Regarding pharmacologic cardioversion, Yealy and colleagues stated that "a calcium channel blocker or $\beta$-adrenergic blocker should be given before a type IA agent to control the ventricular rate." If slowing the ventricular response in RAFF before attempting cardioversion does not improve the success rate, it wastes time and could lead to unnecessary complications. Identifying factors that can positively influence the success of cardioversion will allow more efficacious ED management of RAFF. In those patients who might benefit from rhythm control in the ED, increasing successful conversion may reduce use of anticoagulants, thus avoiding the attendant complications.

\section{GOALS}

The goals of this study were to determine, for electrical and chemical cardioversion separately, 1) whether rate control medication use before cardioversion influences success and 2) if any other factors are independently associated with successful conversion. Chemical and 
electrical cardioversion have significantly different mechanisms of action and therefore were analyzed as separate cohorts.

\section{METHODS}

\section{Study design and setting}

This is a secondary analysis of a previous report describing geographic variability in the management of RAFF ${ }^{4}$ henceforth known as the "RAFF study." The data in the present study were collected by a health records review on a cohort of patients who presented to eight academic EDs from January 1 to December 31, 2008. All centres represent academic hospitals across Canada and have a median annual census of 50,000 patients per year. ${ }^{4}$

\section{Study subjects}

We included patients from the RAFF study if cardioversion was attempted either by chemical or electrical means. Some patients had both chemical and electrical cardioversion attempted. Patients were enrolled in the RAFF study if they were age 18 years or older, with the primary diagnosis of a recent-onset episode (first episode or recurrent) of rapid AF or AFL where symptoms required urgent management and where acute rate control or rhythm control were both management options. Patients were included if they had a clear history of onset within 48 hours or onset within 7 days if adequately anticoagulated (international normalized ratio $>2$ ). Exclusion criteria were 1) permanent $\mathrm{AF}$ or $\mathrm{AFL} ; 2$ ) primary presentation was for another condition, for example, acute coronary syndrome with chest pain or acute electroencephalographic changes, heart failure requiring immediate treatment with intravenous medication or noninvasive ventilation, pneumonia with temperature $>38.5^{\circ} \mathrm{C}$, or pulmonary embolism with chest pain or dyspnea; or 3 ) who were previously enrolled during the study period. The study was approved by the research ethics boards of the respective study hospitals.

\section{Data collection}

The data collection methods were described in the original RAFF study. ${ }^{4}$ A trained research nurse at each site performed the primary case identification and data abstraction. Potential cases were identified from the electronic patient database using key search terms (AF, AFL, arrhythmia, palpitations). The ED charts of these potential cases were reviewed for eligibility by the research nurses. A research nurse at the coordinating centre provided central quality assurance for both case identification and data. The first 25 completed case record forms from a site had to be approved for accuracy of data abstraction before additional cases could be abstracted. The coordinating centre nurse resolved any unclear or ambiguous data elements in consultation with the principal investigator. Interrater reliability was not formally assessed. Data were entered into a database with double entry of key variables. In addition, limited data were collected for patients with a primary complaint of RAFF who had exclusion criteria.

\section{Data analysis}

Baseline characteristics, interventions, and disposition of the study subjects were described with simple percentages, means, medians, and standard deviations where appropriate. The $\mathrm{CHADS}_{2}$ score was dichotomized into zero and nonzero scores to facilitate multivariate analysis. Data on specific medications used for cardioversion were compressed into four categories (procainamide, amiodarone, propafenone, other) due to the sparse distribution in many of the medication types. Univariate analyses were conducted to find associations between factors considered to potentially impact the outcome of interest, successful cardioversion. When variables had significant numbers of missing data, these factors were not considered. Chisquare and Fisher exact tests were used for categorical data and the Wilcoxon rank sum test for continuous data. Patients undergoing chemical and electrical cardioversion were analyzed separately.

We conducted forward stepwise logistic regression (LR) to determine whether use of rate control medication, adjusted for all other available variables, facilitated successful cardioversion. Univariate associations with $p$ values $<0.20$ were offered to the multivariate LR model, with "use of rate control medication" forced into the models. Interaction terms were tested on the electrical cardioversion data set only due to the small number of significant variables in the chemical cardioversion data set. The LR provided adjusted odds ratios (ORs) and 95\% confidence intervals (CIs) associated with successful cardioversion of RAFF. Each final 
model's fit was tested using the Hosmer and Lemeshow goodness-of-fit statistic and the area under the receiver operating characteristic (ROC) curve. ${ }^{10}$ Data analyses were conducted with $S A S$ statistical software version 9.2 (SAS Institute, Inc., Cary, NC).

\section{RESULTS}

\section{Characteristics of study subjects}

The RAFF study included 1,068 patients, of whom 634 (59.4\%) underwent attempted cardioversion and are included in this analysis. Table 1 describes the characteristics of this cohort; 428 patients underwent electrical cardioversion, 354 underwent chemical cardioversion, and 148 received both. It is probable that some who failed drug treatment were cardioverted electrically. The presenting rhythm was AF in $88.6 \%$ and AFL in $11.4 \%$. There were no major differences between the electrical and chemical cardioversion groups. Concomitant serious diagnoses were made in the minority of patients, with heart failure diagnosed in $2.2 \%$ and acute coronary syndrome in $1.4 \%$ of subjects.

\section{Interventions and outcomes}

Table 2 describes the interventions delivered and the disposition of the patients in the cohort. Forty-eight percent of study subjects received rate control medication; however, this was more common in the chemical cardioversion group $(55.7 \%)$ than in the electrical cardioversion group (41.4\%). Procainamide was the most common drug used for cardioversion, followed by amiodarone. Patients cardioverted electrically were discharged home more often $(91.8 \%)$ than those cardioverted chemically (83.3\%). Of note, only $37.0 \%$ in the chemical group actually converted with a drug and $32.8 \%$ needed electrical cardioversion.

Table 1. Baseline characteristics for patients in the electrical and chemical cardioversion cohorts

\begin{tabular}{|c|c|c|c|}
\hline Characteristic & $\begin{array}{l}\text { All patients } \\
(N=634)\end{array}$ & $\begin{array}{l}\text { Cohort receiving electrical } \\
\text { cardioversion }(n=428)\end{array}$ & $\begin{array}{l}\text { Cohort receiving chemical } \\
\text { cardioversion }(n=354)^{*}\end{array}$ \\
\hline Age in years, mean (SD) & $61.1(15.5)$ & $59.1(15.2)$ & $62.5(15.9)$ \\
\hline Range & $18-93$ & $18-93$ & $19-93$ \\
\hline Male, $n(\%)$ & 392 (61.8) & $285(66.6)$ & $212(59.9)$ \\
\hline \multicolumn{4}{|l|}{ Initial rhythm, $n(\%)$} \\
\hline Atrial fibrillation & 562 (88.6) & 372 (86.9) & $323(91.2)$ \\
\hline Atrial flutter & $72(11.4)$ & $56(13.1)$ & $31(8.8)$ \\
\hline Duration of arrhythmia in hours, mean (SD) & $11.3(21.4)$ & $11.4(19.9)$ & $10.8(20.4)$ \\
\hline Range & $0.5-168$ & $1-168$ & $0.5-168$ \\
\hline Initial heart rate, mean (SD) & $123.2(27.8)$ & $123.6(28.3)$ & $122.1(27.3)$ \\
\hline Previous atrial fibrillation, $n(\%)$ & $455(71.8)$ & $311(72.7)$ & $260(73.5)$ \\
\hline $\mathrm{CHADS}_{2}$ score $>0,{ }^{\dagger} n(\%)$ & $336(53.0)$ & $214(50.0)$ & $196(55.4)$ \\
\hline \multicolumn{4}{|l|}{ Other medical history, $n(\%)$} \\
\hline Hypertension & 259 (40.9) & $171(40.0)$ & $145(41.0)$ \\
\hline Valvular heart disease & $47(7.4)$ & $31(7.2)$ & $28(7.9)$ \\
\hline Pacemaker/ICD & $16(2.5)$ & $8(1.9)$ & $12(3.4)$ \\
\hline COPD/asthma & $48(7.6)$ & $32(7.5)$ & $27(7.6)$ \\
\hline \multicolumn{4}{|l|}{ Current medications, $n(\%)$} \\
\hline$\beta$-Blockers & $210(33.1)$ & $139(32.5)$ & $126(35.6)$ \\
\hline Calcium channel blocker & $102(16.1)$ & $67(15.7)$ & $57(16.1)$ \\
\hline Sotalol & $45(7.1)$ & $33(7.7)$ & $19(5.4)$ \\
\hline Amiodarone & $28(4.4)$ & $20(4.7)$ & $12(3.4)$ \\
\hline Digoxin & $20(3.2)$ & $10(2.3)$ & $14(4.0)$ \\
\hline Procainamide & $1(0.2)$ & $1(0.2)$ & $0(0.0)$ \\
\hline \multicolumn{4}{|l|}{ Other conditions identified while in ED, $n(\%)$} \\
\hline Congestive heart failure & $14(2.2)$ & $8(1.9)$ & $7(2.0)$ \\
\hline Acute coronary syndrome & $9(1.4)$ & $5(1.2)$ & $5(1.4)$ \\
\hline
\end{tabular}




\begin{tabular}{|c|c|c|c|}
\hline Characteristic & $\begin{array}{l}\text { All patients } \\
(N=634)\end{array}$ & $\begin{array}{l}\text { Patients receiving electrical } \\
\text { cardioversion }(n=428)\end{array}$ & $\begin{array}{l}\text { Patients receiving chemical } \\
\text { cardioversion }(n=354)^{*}\end{array}$ \\
\hline Rate control medications given, $n(\%)$ & $304(48.0)$ & $177(41.4)$ & $197(55.7)$ \\
\hline Metoprolol & $200(18.7)$ & $124(29.0)$ & $122(34.5)$ \\
\hline Diltiazem & $100(9.4)$ & $52(12.1)$ & $71(20.1)$ \\
\hline Verapamil & $3(0.3)$ & $1(0.2)$ & $3(0.8)$ \\
\hline Other & $1(0.1)$ & $0(0.0)$ & $1(0.3)$ \\
\hline Drug given for conversion, $n(\%)$ & $354(55.8)$ & $148(34.6)$ & $354(100.0)$ \\
\hline Procainamide & $220(34.7)$ & $95(22.2)$ & $220(62.1)$ \\
\hline Amiodarone & $53(8.4)$ & $30(7.0)$ & $53(15.0)$ \\
\hline Propafenone & $30(4.7)$ & $7(1.6)$ & $30(8.5)$ \\
\hline Other & $51(8.0)$ & $16(3.7)$ & $51(14.4)$ \\
\hline \multicolumn{4}{|l|}{ Disposition, $n(\%)$} \\
\hline Discharged home & $558(88.0)$ & 91.8 & 83.3 \\
\hline Next-day ED follow-up & $15(2.7)$ & 0.5 & 4.8 \\
\hline $\begin{array}{l}\text { Converted to sinus rhythm prior to ED discharge, } \\
n(\%)\end{array}$ & $545(86.0)$ & 92.8 & 79.1 \\
\hline Electrical & $377(59.5)$ & 88.1 & 32.8 \\
\hline Spontaneous & $36(5.7)$ & 1.6 & 9.3 \\
\hline Not converted & $89(14.0)$ & 7.2 & 20.9 \\
\hline Drug & $132(20.8)$ & 3.0 & 37.0 \\
\hline Heart rate prior to discharge, mean (bpm) & 76.1 & 73.7 & 78.0 \\
\hline ED length of stay, median (hours) & 6.4 & 5.1 & 6.1 \\
\hline
\end{tabular}

\section{Factors associated with successful electrical cardioversion}

The factors analyzed for influence on successful electrical cardioversion are listed in Table 3. Age tended to significance, with younger patients more likely to be successfully cardioverted than older patients. Patients with hypertension were also less likely to be converted. A $\mathrm{CHADS}_{2}$ score $>0$ was associated with decreased conversion success. The two strongest negative associations were use of rate control and rhythm control medications. Sixty-four percent of those failing electrical cardioversion had their ventricular rate slowed before the attempt versus $38.4 \%$ of those successfully converted $(p<0.001)$. Similarly, $62.0 \%$ in the group failing conversion already underwent attempted chemical conversion versus $31.0 \%$ in the group successfully cardioverted $(p<0.0001)$. Contrary to other reports, ${ }^{7}$ use of anteroposterior pad position made little difference, although the number with complete information (150) was small.

Multivariate analysis showed that after adjustment, using rate control was associated with decreased likelihood of successful conversion (OR 0.39, 95\% CI
0.21-0.74). Attempted chemical conversion also remained significant, with an OR of 0.28 (95\% CI 0.15-0.53). Interestingly, a $\mathrm{CHADS}_{2}$ score $>0$ also predicted against success, with an OR of $0.43(95 \%$ $0.23-0.83)$. For the logistic regression model, the Hosmer and Lemeshow goodness-of-fit statistic was $\chi 2=1.49, p=0.960$, and area under the ROC curve $c$ $=0.733$.

\section{Factors associated with successful chemical cardioversion}

Table 4 describes the chemical cardioversion cohort. Those who were successfully cardioverted tended to be older (mean age 66 versus 60 ) and less frequently male (46.3\% versus $68.4 \%$ ). In contrast to the electrical cohort, they also tended to have a higher proportion of nonzero $\mathrm{CHADS}_{2}$ scores $(61.0 \%)$ than the group that failed $(51.8 \%)$; however, this did not reach significance $(p=0.08)$. There were striking differences in the success rates between different drugs. Procainamide was used in $74.3 \%$ of successful cardioversions versus $54.6 \%$ of unsuccessful conversions. The next most commonly used drug, amiodarone, was used in 


\begin{tabular}{|c|c|c|c|}
\hline Factor & $\begin{array}{l}\text { Successful } \\
\text { conversion } \\
(n=378)\end{array}$ & $\begin{array}{l}\text { Failed conversion } \\
\qquad(n=50)\end{array}$ & $p$ value \\
\hline Age in years (mean) & 58.5 & 63.6 & 0.06 \\
\hline Male sex $(\%)$ & 66.4 & 68.0 & 0.82 \\
\hline \multicolumn{4}{|l|}{ Past medical history (\%) } \\
\hline Hypertension & 38.4 & 52.0 & 0.06 \\
\hline Valvular heart disease & 6.9 & 10.0 & 0.39 \\
\hline Pacemaker/ICD & 2.1 & 0.0 & 0.60 \\
\hline COPD/asthma & 7.1 & 10.0 & 0.40 \\
\hline Hours since onset (median) & 4.0 & 4.0 & 0.61 \\
\hline Prior episode AF (\%) & 71.4 & 82.0 & 0.12 \\
\hline Prior ablation ( $n=232 ; 34)$ & 12.9 & 14.7 & 0.79 \\
\hline Prior electrical cardioversion ( $n=200 ; 27$ ) & 79.0 & 70.4 & 0.31 \\
\hline Prior chemical cardioversion ( $n=121 ; 18)$ & 44.6 & 38.9 & 0.65 \\
\hline $\mathrm{CHADS}_{2}$ score $>0(\%)$ & 47.6 & 68.0 & 0.01 \\
\hline \multicolumn{4}{|l|}{ Current medication use (\%) } \\
\hline$\beta$-Blocker & 31.2 & 42.0 & 0.13 \\
\hline Sotalol & 7.9 & 6.0 & 0.78 \\
\hline Amiodarone & 4.8 & 4.0 & 1.00 \\
\hline Procainamide & 0.3 & 0.0 & 1.00 \\
\hline Digoxin & 2.1 & 4.0 & 0.33 \\
\hline Initial rhythm atrial flutter (\%) & 12.7 & 16.0 & 0.52 \\
\hline Chemical cardioversion attempted (\%) & 31.0 & 62.0 & $<0.0001$ \\
\hline Rate control used in ED (\%) & 38.4 & 64.0 & $<0.001$ \\
\hline Initial heart rate (mean) & 124 & 123 & 0.98 \\
\hline AP pad position $(n=132,18)(\%)$ & 72.7 & 61.1 & 0.31 \\
\hline
\end{tabular}

$8.1 \%$ of successful and $19.3 \%$ of unsuccessful conversions.

Multivariate analysis of the chemical cardioversion cohort showed that use of rate control medication had no impact on successful cardioversion, after adjusting for other factors (OR 1.29; 95\% CI 0.82-2.03). Female sex (OR 2.37; 95\% CI 1.50-3.72) and use of procainamide (OR 2.32; 95\% CI 1.43-3.74) were significantly associated with successful conversion. For the logistic regression model, the Hosmer and Lemeshow goodness-of-fit statistic was $\chi 2=3.16$, $p$ $=0.788$, and area under the ROC curve $\mathrm{c}=0.667$.

\section{LIMITATIONS}

The observational nature of this study means that the associations discovered may not be causal and should be interpreted with caution. A small number of the variables had a significant amount of missing data (e.g., pad position in those cardioverted electrically) and could not be included in the LR model; thus, significant relationships may not have been detected. Associations tested were limited by the data set collected; thus, some previously described factors predictive of successful cardioversion (e.g., body weight, body mass index, comorbidities) were unavailable. Adverse events were not specifically investigated in this study. Finally, this study was not designed to test longer-term outcomes, for example, stroke rate after discharge, rate of relapse back to the ED, and patient satisfaction with the respective approaches, or to determine the optimal management strategy.

\section{DISCUSSION}

\section{Summary and interpretation of all findings}

To our knowledge, this is the first article to challenge the theory that slowing the ventricular rate in RAFF enhances cardioversion success. When used before 


\begin{tabular}{|c|c|c|c|}
\hline Factor & $\begin{array}{c}\text { Successful } \\
\text { conversion } \\
(n=136)\end{array}$ & $\begin{array}{l}\text { Failed conversion } \\
\qquad(n=218)\end{array}$ & $p$ value \\
\hline Age in years (mean) & 66 & 60 & 0.01 \\
\hline Male sex (\%) & 46.3 & 68.4 & $<0.0001$ \\
\hline \multicolumn{4}{|l|}{ Past medical history (\%) } \\
\hline Hypertension & 41.2 & 40.8 & 0.95 \\
\hline Valvular heart disease & 8.1 & 7.8 & 0.92 \\
\hline Pacemaker/ICD & 2.2 & 4.1 & 0.38 \\
\hline COPD/asthma & 10.3 & 6.0 & 0.14 \\
\hline $\mathrm{CHADS}_{2}$ score $>0(\%)$ & 61.0 & 51.8 & 0.09 \\
\hline Prior episode AF (\%) & 70.6 & 75.2 & 0.34 \\
\hline \multicolumn{4}{|l|}{ Current medication use (\%) } \\
\hline$\beta$-Blocker & 37.5 & 34.4 & 0.55 \\
\hline Sotalol & 5.9 & 5.1 & 0.73 \\
\hline Amiodarone & 3.7 & 3.2 & 1.00 \\
\hline Procainamide & NA & NA & NA \\
\hline Digoxin & 5.2 & 3.2 & 0.36 \\
\hline Median hours since onset & 3.0 & 5.0 & 0.04 \\
\hline Associated CHF (\%) & 0.7 & 2.8 & 0.26 \\
\hline Associated ACS (\%) & 2.2 & 0.9 & 0.38 \\
\hline Initial rhythm atrial flutter (\%) & 5.2 & 11.0 & 0.06 \\
\hline Rate control used in ED (\%) & 61.0 & 52.3 & 0.11 \\
\hline \multicolumn{4}{|l|}{ Agent used for conversion (\%) } \\
\hline Procainamide & 74.3 & 54.6 & 0.0002 \\
\hline Amiodarone & 8.1 & 19.3 & 0.0041 \\
\hline Propafenone & 8.8 & 8.3 & 0.85 \\
\hline Other & 2.9 & 3.7 & 0.77 \\
\hline Initial heart rate, bpm & 125.5 & 120 & 0.11 \\
\hline
\end{tabular}

chemical cardioversion, it made no difference in the proportion that converted; when used before electrical cardioversion, it reduced it. Of interest, when rhythm control drugs were used on people who then had electrical cardioversion, the procedure was significantly less likely to be successful. It is possible that the pharmacodynamics of the drugs alter the myocardium such that it becomes resistant to electrical cardioversion. Alternatively, the explanation could be that those patients who fail chemical cardioversion have an intrinsic resistance to any form of cardioversion such that they are preselected to fail the electrical attempt. Patients with a nonzero $\mathrm{CHADS}_{2}$ score more often failed electrical cardioversion even after adjustment for other factors. This finding fits with the fact that the components that form the score are themselves risk factors for heart disease, and "prior transient ischemic attack or stroke" is a proxy for atherosclerosis, a contributor to heart disease. It is not suggested that cardioversion be avoided in these patients as $61 \%$ of the study cohort was in this category. In this study, we did not find the duration of symptoms to be a predictor of successful cardioversion after adjusting for other factors.

Additional interesting results arose from the chemical multivariate analysis. Slowing the ventricular rate before attempting conversion had no impact on the success rate, in contrast to electrical cardioversion. Unexpectedly, males were significantly less likely than their female counterparts to be successfully chemically cardioverted. In this cohort, the most frequently used agent, procainamide, was the only one to be significantly associated with successful conversion. The next most commonly used drug in this study, amiodarone, despite predicting against successful conversion in univariate analysis, was not a significant factor in multivariate analysis. 


\section{Comparison to prior studies}

Previous studies that examined predictors of immediate success of cardioversion come from the cardiology literature, which focus on longer-term ( $>48$ hours) dysrhythmias and use single-strategy cardioversion (i.e., drug or direct current). A study using esmolol prior to ibutilide cardioversion showed that it improved the odds of conversion ${ }^{11}$; however, this was a small, nonblinded study that excluded patients with $\mathrm{AF}$ duration $<24$ hours and thus has limited applicability to the ED population. Evidence is mixed on whether using antiarrhythmics prior to electrical cardioversion influences success. Amiodarone and sotalol use have been shown to increase successful electrical cardioversion compared to no drug therapy. ${ }^{12}$ In another study, pretreatment with amiodarone had no impact on the immediate success of electrical cardioversion. ${ }^{13}$ These studies used medication for weeks before planned cardioversion of dysrhymias of duration anywhere up to 1 year. Studies have found increasing symptom duration to be a negative predictor of successful cardioversion..$^{14,15}$ This was not confirmed in our study, perhaps because patients presented relatively early, before any difference would have been found.

To our knowledge, there have been no previous trials examining the primary research question of whether controlling the ventricular rate before ED cardioversion affects success. In a small study, electrical cardioversion was successful in 7 of 10 patients pretreated with digoxin for 48 hours versus 7 of 7 treated with amiodarone and 3 of 3 with sotalol. ${ }^{16}$ The unexpected finding that female sex was a predictor of successful conversion was shown previously in an Italian study. ${ }^{17}$ Use of procainamide to convert RAFF was recently shown to have a success rate of $58 \%$ in an observational study. ${ }^{18}$ When used in a protocol together with electrical cardioversion as a backup, the adverse event rate was $7.6 \%$, although the majority of this was attributed to sedation-related hypotension. Despite being the medication most strongly associated with success in this cohort, procainamide is classed as IIb ("less effective") in the ACC/AHA/ESC practice guidelines. ${ }^{5}$ Previous studies using amiodarone have had mixed results; evidence exists showing that it is no more effective than placebo, is slower than other drugs in achieving sinus rhythm, and has higher adverse event rates. ${ }^{19-25}$

\section{Summary of study strengths}

This study is the first to answer the question of whether rate control is an effective strategy before cardioversion in RAFF. The relatively large number of patients included, representing eight Canadian EDs with different protocols for managing RAFF, enhances its external validity.

\section{Clinical implications}

This study failed to identify an advantage in slowing the ventricular rate before cardioverting RAFF and is a practice best avoided unless cardioversion is delayed, for example, for transesophageal echocardiography. Considering the strong association between use of procainamide and successful conversion, the lack of a similar relationship with amiodarone, and previous studies showing its lack of efficacy, use of amiodarone in converting RAFF in the ED should be discouraged.

\section{Research implications}

Future research should be directed toward comparative effectiveness studies in RAFF instead of single treatments, to determine whether a strategy of "drug firstelectricity later" is optimal, and to determine meaningful clinical outcomes and adverse event rates.

\section{CONCLUSION}

Use of rate control medication in RAFF before electrical cardioversion reduces efficacy and is illadvised. Use of procainamide, and not amiodarone, in chemical cardioversion is strongly associated with success and is recommended. Future research should be directed to prospective, randomized trials to determine the success rate in the ED of the various drugs and protocols in attaining sinus rhythm in RAFF and their respective rate of adverse events.

Competing interests: None declared.

\section{REFERENCES}

1. Feinberg WM, Blackshear JL, Laupacis A, et al. Prevalence, age distribution, and gender of patients with atrial fibrillation. Analysis and implications. Arch Intern Med 1995;155: 469-73, doi:10.1001/archinte.1995.00430050045005.

2. Raitt MH, Volgman AS, Zoble RG, et al. Prediction of the recurrence of atrial fibrillation after cardioversion in the 
Atrial Fibrillation Follow-up Investigation of Rhythm Management (AFFIRM) study. Am Heart 7 2006;151:3906, doi:10.1016/j.ahj.2005.03.019.

3. Roy D, Talajic M, Nattel S, et al. Rhythm control versus rate control for atrial fibrillation and heart failure. $N$ Engl 7 Med 2008;358:2667-77, doi:10.1056/NEJMoa0708789.

4. Stiell IG, Clement CM, Brison RJ, et al. Variation in management of recent-onset atrial fibrillation and flutter among academic hospital emergency departments. Ann Emerg Med, 201157:13-21.

5. Fuster V, Ryden LE, Cannom DS, et al. ACC/AHA/ESC 2006 Guidelines for the Management of Patients with Atrial Fibrillation: a report of the American College of Cardiology/ American Heart Association Task Force on Practice Guidelines and the European Society of Cardiology Committee for Practice Guidelines (Writing Committee to Revise the 2001 Guidelines for the Management of Patients With Atrial Fibrillation): developed in collaboration with the European Heart Rhythm Association and the Heart Rhythm Society. Circulation 2006;114:e257-354, doi:10.1161/ CIRCULATIONAHA.106.177292.

6. Blich M, Edoute Y. Electrical cardioversion for persistent or chronic atrial fibrillation: outcome and clinical factors predicting short and long term success rate. Int 7 Cardiol 2006;107:389-94, doi:10.1016/j.ijcard.2005.03.057.

7. Reiffel JA. Cardioversion for atrial fibrillation: treatment options and advances. Pacing Clin Electrophysiol 2009;32: 1073-84, doi:10.1111/j.1540-8159.2009.02441.x.

8. Hagens VE, Crijns HJ, Van Veldhuisen DJ, et al. Rate control versus rhythm control for patients with persistent atrial fibrillation with mild to moderate heart failure: results from the RAte Control versus Electrical cardioversion (RACE) study. Am Heart 7 2005;149:1106-11, doi:10.1016/ j.ahj.2004.11.030.

9. Yealy D, Delbridge T. Dysrhythmias. In: Marx J, Hockberger R, Walls R, et al, editors, Rosen's emergency medicine: concepts and clinical practice. 7 th ed. Philadelphia: Mosby Elsevier; 2010. p. 984-1024.

10. Hosmer DW, Hosmer T, Le Cessie S, Lemeshow S. A comparison of goodness-of-fit tests for the logistic regression model. Stat Med 1997;16:965-80, doi:10.1002/ (SICI)1097-0258(19970515)16:9<965::AID-SIM509>3.0. $\mathrm{CO} ; 2-\mathrm{O}$.

11. Fragakis N, Bikias A, Delithanasis I, et al. Acute betaadrenoceptor blockade improves efficacy of ibutilide in conversion of atrial fibrillation with a rapid ventricular rate. Europace 2009;11:70-4, doi:10.1093/europace/eun302.

12. Vijayalakshmi K, Whittaker VJ, Sutton A, et al. A randomized trial of prophylactic antiarrhythmic agents (amiodarone and sotalol) in patients with atrial fibrillation for whom direct current cardioversion is planned. Am Heart 7 2006;151:863.e1-e6, doi:10.1016/j.ahj.2005.09.009.

13. Channer KS, Birchall A, Steeds RP, et al. A randomized placebo-controlled trial of pre-treatment and short- or long-term maintenance therapy with amiodarone supporting DC cardioversion for persistent atrial fibrillation. Eur Heart 7 2004;25:144-50, doi:10.1016/j.ehj.2003.10.020.

14. Ergene U, Ergene O, Cete Y, et al. Predictors of success in the conversion of new-onset atrial fibrillation using oral propafenone. Eur 7 Emerg Med 1998;5:425-8, doi:10.1097/ 00063110-199812000-00008.

15. Bianconi L, Boccadamo R, Pappalardo A, et al. Effectiveness of intravenous propafenone for conversion of atrial fibrillation and flutter of recent onset. Am 7 Cardiol 1989;64:335-8, doi:10.1016/0002-9149(89)90530-4.

16. Joseph AP, Ward MR. A prospective, randomized controlled trial comparing the efficacy and safety of sotalol, amiodarone, and digoxin for the reversion of new-onset atrial fibrillation. Ann Emerg Med 2000;36:1-9, doi:10.1067/ mem.2000.107655.

17. Rostagno C, Bacci F, Paladini B, et al. Predictors of successful at-home chemical cardioversion in new-onset atrial fibrillation. Eur 7 Emerg Med 1994;1:131-5.

18. Stiell IG, Clement CM, Perry JJ, et al. Association of the Ottawa Aggressive Protocol with rapid discharge of emergency department patients with recent-onset atrial fibrillation or flutter. CFEM 2010;12:181-91.

19. Martinez-Marcos FJ, Garcia-Garmendia JL, OrtegaCarpio A, et al. Comparison of intravenous flecainide, propafenone, and amiodarone for conversion of acute atrial fibrillation to sinus rhythm. Am 7 Cardiol 2000;86:950-3, doi:10.1016/S0002-9149(00)01128-0.

20. Tijunelis MA, Herbert ME. Myth: Intravenous amiodarone is safe in patients with atrial fibrillation and WolffParkinson-White syndrome in the emergency department. CFEM 2005;7:262-5.

21. Hilleman DE, Spinler SA. Conversion of recent-onset atrial fibrillation with intravenous amiodarone: a meta-analysis of randomized controlled trials. Pharmacotherapy 2002;22:6674, doi:10.1592/phco.22.1.66.33492.

22. Chevalier P, Durand-Dubief A, Burri H, et al. Amiodarone versus placebo and class Ic drugs for cardioversion of recentonset atrial fibrillation: a meta-analysis. $7 \mathrm{Am}$ Coll Cardiol 2003;41:255-62, doi:10.1016/S0735-1097(02)02705-5.

23. Letelier LM, Udol K, Ena J, et al. Effectiveness of amiodarone for conversion of atrial fibrillation to sinus rhythm: a meta-analysis. Arch Intern Med 2003;163:777-85, doi:10.1001/archinte.163.7.777.

24. Miller MR, McNamara RL, Segal JB, et al. Efficacy of agents for pharmacologic conversion of atrial fibrillation and subsequent maintenance of sinus rhythm: a meta-analysis of clinical trials. 7 Fam Pract 2000;49:1033-46.

25. Thomas SP, Guy D, Wallace E, et al. Rapid loading of sotalol or amiodarone for management of recent onset symptomatic atrial fibrillation: a randomized, digoxincontrolled trial. Am Heart $f$ 2004;147:E3, doi:10.1016/ S0002-8703(03)00526-X. 